\title{
Brasil: Crisis financiera internacional y políticas anticíclicas
}

\author{
Tito Belchior Silva Moreira y \\ Fernando Antônio Ribeiro Soares
}

RESUMEN

En este trabajo se evalúa la eficacia de las medidas anticíclicas adoptadas por el gobierno brasileño para mitigar los efectos de la crisis de las hipotecas de alto riesgo. En este contexto, se analizan las repercusiones de las políticas monetaria, fiscal y crediticia en algunos de los principales agregados macroeconómicos. El análisis empírico reveló que la política crediticia expansionista fue un factor decisivo para aumentar el consumo de las familias y el producto agregado durante el período de la crisis. Mientras que la política monetaria expansionista también fue importante para incrementar el producto agregado durante dicho período, los gastos relativos a las inversiones no resultaron sensibles a las políticas anticíclicas.

PALABRAS CLAVE

CLASIFICACIÓN JEL

AUTORES
Crisis financiera, condiciones económicas, macroeconomía, Brasil, política económica, ciclos económicos, política monetaria, política fiscal, política crediticia, indicadores económicos

O54, E63, E65

Tito Belchior Silva Moreira. Profesor/investigador del Departamento de Economía de la Universidad Católica de Brasilia. tito@pos.ucb.br

Fernando Antônio Ribeiro Soares. Asesor de la Secretaría Ejecutiva del Ministerio de Hacienda del Brasil. fernando.a.r.soares@gmail.com 
I

\section{Introducción}

A comienzos de la década de 2000, pero sobre todo entre 2003 y 2007, la economía mundial presentaba un marcado dinamismo, acompañado de altas tasas de crecimiento económico y estabilidad de los precios, de manera tal que se creía que los gobiernos habían dominado los ciclos económicos.

Sin embargo, si bien el panorama descrito anteriormente refleja una época de gran evolución en la economía mundial, esta y en particular la economía estadounidense comenzaron a presentar evidentes síntomas de fragilidad financiera a partir de 2007. Ese año se inicia un proceso de gran desestabilización de los mercados, especialmente del mercado inmobiliario.

Los desequilibrios en el mercado inmobiliario y de activos en general obedecieron tanto a factores macroeconómicos como a aspectos inherentes al mercado financiero. En los Estados Unidos de América, polo inductor de la crisis mundial, el elevado "desahorro" -reconocido a partir de los extraordinarios déficits en la cuenta de transacciones corrientes-y la política monetaria excesivamente expansionista provocaron un marcado proceso de apalancamiento financiero, reforzado por la "desregulación" bancaria y el surgimiento de diversas innovaciones financieras, entre ellas los derivados de crédito y la desmedida titulización.

Ese proceso se agudizó y el apalancamiento de empresas y familias creció de manera exponencial. En 2007 el endeudamiento asintótico llegó a su límite. Al primer síntoma de la fragilidad financiera de los bancos y de la excesiva exposición a deudores con insuficiente capacidad de pago, los acreedores realizaron peticiones de reposición del margen de garantía y suspendieron la refinanciación de las deudas. Se produjo entonces una corrida bancaria, con un potencial capaz de afectar seriamente la liquidez del sistema financiero o incluso volverlo insolvente. En esas circunstancias, las autoridades monetarias y económicas de todo el mundo comenzaron a realizar operaciones de rescate y salvamento. En principio, el objetivo de tales operaciones no era mitigar la desaceleración de la actividad económica, sino evitar el colapso financiero.

Los efectos de la crisis financiera no tardaron en pasar al "lado real" de la economía. Si bien el principal canal de transmisión de la crisis fueron los mercados de crédito, tanto accionario como bancario, el deterioro de las expectativas también desempeñó un papel importante. Se registró así una pronunciada reducción de las corrientes de comercio y de capitales a nivel mundial y se pospusieron las decisiones de inversión. Esto se tradujo en el congelamiento de la demanda agregada.

Las repercusiones de la crisis en la economía brasileña se sintieron sobre todo a partir del segundo semestre de 2008, en especial durante el último trimestre de ese ejercicio, y se manifestaron de la siguiente manera: i) contracción del crédito nacional y mundial; ii) destrucción de riqueza como resultado de la declinación del precio de los activos; iii) deterioro de las expectativas; iv) vertiginosa caída de los precios de los productos básicos, y v) reducción del comercio mundial.

Aunque un poco tarde - pues, como se señaló, los efectos de la crisis mundial empezaron a advertirse en 2007-, las autoridades gubernamentales brasileñas comenzaron a implementar una serie de medidas para enfrentar el deterioro del contexto económico, tanto de carácter general (horizontales) como específico o sectorial (verticales) ${ }^{1}$. Se procuraba de esa forma mitigar el impacto de la crisis internacional en el Brasil, evitando una brusca desaceleración de la economía y sus negativas consecuencias en el producto y el empleo.

En ese marco, se adoptaron políticas anticíclicas fiscales, crediticias y monetarias. Con respecto de algunos sectores específicos de la economía, la política fiscal implementó la reducción del impuesto sobre los productos industrializados (IPI). La política crediticia se estableció principalmente a partir de bancos e instituciones financieras públicas, que suplieron la falta de crédito privado en varios sectores de la economía, entre ellos el de la construcción civil y el automotor. Por último, la política monetaria supuso una considerable disminución de la tasa de interés básica de la economía, que se reflejó tanto en la tasa nominal como en la tasa real, hecho que demuestra el predominio del aspecto monetario en la conducción de la política económica brasileña.

\footnotetext{
${ }^{1}$ Las medidas horizontales son aquellas de carácter macroeconómico que, por lo tanto, afectan a la sociedad como un todo. En este caso, se puede citar la reducción de la tasa de interés básica de la economía. Las verticales, por su parte, son específicas y afectan a determinados sectores productivos, como por ejemplo, el sector automotor o el de la construcción civil.
} 
En este trabajo no se abordan los orígenes o las explicaciones de los factores determinantes de la crisis del sistema financiero estadounidense y sus repercusiones en la economía mundial. Varios autores se ocupan de tales aspectos, entre ellos Mohan (2009); Rose y Spiegel (2009); Taylor (2009); Kindleberger y Aliber (2009); Krugman (2009); Lopes (2009); Rogoff y Reinhart (2010); Roubini y Mihm (2010); Kacef y López-Monti (2010). La cronología de la crisis también excede el objetivo del presente trabajo, pero puede verse en Torres Filho (2008) y Torres Filho y Rodrigues Borça Júnior (2008). El núcleo de este análisis consiste en la evaluación de las medidas anticíclicas adoptadas por el gobierno brasileño para mitigar los efectos de la crisis. En ese contexto, se analizan las repercusiones de las políticas monetaria, fiscal y crediticia en algunos de los principales agregados macroeconómicos y en determinados sectores de la economía que recibieron incentivos gubernamentales.

Luego de estas consideraciones, se detalla a continuación la estructura del trabajo. Después de esta breve Introducción, en la sección II se presentan algunos aspectos metodológicos, con énfasis en las bases de datos, y se describen las principales medidas anticíclicas adoptadas en el Brasil. En la sección III se construyen estimaciones de los efectos de las medidas anticíclicas implementadas en la demanda agregada y sus componentes y, por último, en la sección IV se presentan las conclusiones.

\section{II}

\section{Aspectos metodológicos y medidas anticíclicas adoptadas en el Brasil}

Las variables y las respectivas nomenclaturas empleadas en este trabajo (entre paréntesis) son las siguientes: consumo final de las familias, denominado (Cons $s^{\text {fam }}$ ); producto interno bruto a precios básicos, denominado $(Y)$; crédito al sector privado, denominado (Credpri); tasa básica de interés operada por medio del Sistema Especial de Liquidación y Custodia (SELIC) en porcentajes, denominada $(R)$; formación bruta de capital fijo, denominada $(F B C F)$; tasa de inflación según el Î́ndice Nacional de Precios al Consumidor Amplio (IPCA), denominada $(\pi)$; impuestos sobre los productos, denominados (IMP); y producto interno bruto real a precios de mercado deflactado por el Índice General de Precios - Disponibilidades Internas (IGP-DI), denominado (PIB). En el cuadro A.1 del anexo se detallan las variables utilizadas y se especifican las fuentes y unidades de medida.

Las variables citadas se modificaron para volverlas estacionarias y evitar de esa forma regresiones espurias. Las variables consumo final de las familias, crédito al sector privado y formación bruta de capital fijo (inversión) se normalizaron por el PIB a precios básicos. Asimismo, se transformaron en tasas de variación y se volvieron a nombrar, respectivamente, $d\left(\operatorname{Cons}_{t}^{\mathrm{fam}} / Y_{t}\right), d\left(\operatorname{Cred}_{t}^{\text {pri }} / Y_{t}\right)$ y $d\left(F B C F_{t} / Y_{t}\right)^{2}$.

\footnotetext{
2 Admitiendo que $\left(\right.$ Cons $\left._{t}^{\text {fam }} / Y_{t}\right)=a_{t}$, entonces $d\left(\right.$ Cons $\left._{t}^{\text {fam }} / Y_{t}\right)=$ $\left(a_{t}-a_{t-1}\right) / a_{t-1}$.
}

La tasa de interés real (ex post) se calculó como la diferencia entre la tasa SELIC y el IPCA y se denominó $r_{t}$. No se utilizó el valor esperado del IPCA debido a que esa serie solo estuvo disponible a partir de 1999, cuando comenzó el régimen de metas inflacionarias. La variable tasa de interés real también se transformó en tasa de variación y se representó mediante $d\left(r_{t}\right)$.

Para calcular la brecha del producto, denominada Hiato $_{t}^{P I B}$, se utilizó el filtro de Hodrick-Prescott. Este se definió como la diferencia entre PIB real y PIB potencial (trend). Un valor positivo indica exceso de demanda. En forma análoga, un valor negativo representa capacidad ociosa en la utilización de los factores de producción. También se midieron otras variables sobre la base del filtro de Hodrick-Prescott. La brecha de la tasa de interés real, denominada Hiato $_{t}^{r}$, se definió como la diferencia entre la tasa de interés real y la tendencia. Un valor positivo indica una política monetaria restrictiva y, en el caso contrario, una política expansionista.

La brecha de la relación entre impuestos e ingresos $\left(\operatorname{IMP}_{t} / Y_{t}\right)$, denotada por Hiato $t_{t}^{\text {imp } / Y}$, se definió como la diferencia entre esa variable y su tendencia. Un valor positivo indica una política fiscal restrictiva. La brecha de la relación entre crédito concedido al sector privado e ingresos $\left(\operatorname{Cred}_{t}^{\text {pri }} / Y_{t}\right)$, presentada como Hiato ${ }_{t}^{\text {cred }}$, se definió como la diferencia entre $\left(\operatorname{Cred}_{t}^{p r i} / Y_{t}\right)$ y su tendencia. Un valor positivo indica una política crediticia expansionista. Las variables sectoriales también 
se transformaron en brecha y se diferenciaron de sus respectivas tendencias.

Se utilizó el cuadrado de algunas variables, entre ellas $\left(I M P_{t} / Y_{t}\right)^{2},\left(\operatorname{Cred}_{t} / Y_{t}\right)^{2}$ y $\left(I P I_{t} / P_{t}\right)^{2}$, con el objetivo de verificar la existencia de una relación no lineal entre esas variables y la variable dependiente. También se utilizaron dos variables ficticias. La variable ficticia $D 1$, que se extiende desde el primer trimestre de 1991 al segundo trimestre de 1994, distingue al período anterior al Plan Real —caracterizado por la inestabilidad inflacionaria— del período posterior, marcado precisamente por el control del proceso inflacionario. La variable ficticia D2 se utiliza para determinar el período de la crisis de las hipotecas de alto riesgo, que abarca desde el primer trimestre de 2007 hasta el primer trimestre de $2010^{3}$.

En la próxima subsección se analiza la eficacia de las medidas anticíclicas implementadas por el gobierno durante la crisis de las hipotecas de alto riesgo. Para ello se realizan pruebas empíricas mediante los siguientes procedimientos: i) se verifica si las variables utilizadas en los modelos econométricos son estacionarias para evitar la presencia de regresiones espurias; ii) confirmada la "estacionariedad" de las series por medio de pruebas de raíz unitaria — prueba de Dickey-Fuller aumentada y prueba de Phillips-Perron-, se estiman las ecuaciones de mínimos cuadrados ordinarios (MCO); iii) se analizan los residuos mediante un correlograma y se ajustan los errores mediante el método Jarque-Bera con el objetivo de ajustar el término estocástico en forma de ruido blanco, y iv) se interpretan los resultados de las ecuaciones estimadas teniendo en cuenta los signos y la significancia de los parámetros estimados.

\section{Medidas anticíclicas en el Brasil}

Desde la profundización de la crisis financiera mundial, el Gobierno Federal tomó diversas medidas para contener los efectos de la turbulencia internacional en la economía brasileña. A partir de septiembre de 2008, se hizo frente a los graves desequilibrios en el mercado financiero mediante acciones como la subasta de dólares realizada por el Banco Central del Brasil. En otras palabras, la gran fuga de capitales dio lugar a una considerable depreciación, que se atenuó mediante la venta de dólares por parte del banco central. Asimismo, se crearon nuevas líneas de crédito y se aumentaron los límites de financiamiento para intentar

\footnotetext{
${ }^{3}$ La variable ficticia $D 1$ asume los siguientes valores: enero de 1991 a febrero de $1994=1$, y en caso contrario $=0$. La variable ficticia $D 2$, por otra parte, asume el valor de 1 con respecto al período de enero de 2007 a enero de 2010, y en caso contrario $=0$.
}

garantizar la continuidad de la actividad económica del país. También se crearon líneas de financiamiento al consumo, se firmaron decretos para garantizar la estructura del sector bancario y se modificaron las reglas del cobro de los depósitos obligatorios.

Durante la crisis, el gobierno brasileño adoptó políticas económicas anticíclicas que fueron más allá de medidas específicas para atenuar el impacto en determinados sectores. Desde el punto de vista de la política monetaria y crediticia, se destacan la reducción de las reservas bancarias obligatorias, los cortes de la tasa anual de interés básica calculada diariamente por el Sistema Especial de Liquidación y Custodia (SELIC) y el aumento de la oferta de crédito por parte de los bancos públicos. Con esas medidas se procuraba atenuar los efectos negativos de la crisis en la inversión y el consumo a partir de menores tasas de interés y mayor disponibilidad de crédito. Desde el punto de vista fiscal, se destacan la disminución de algunos impuestos y de la meta de superávit primario.

El objetivo de ambas políticas, fiscal y monetaria, era cambiar las expectativas pesimistas y aminorar sus mencionados efectos negativos en el consumo y la inversión. Por último, se procuraba evitar un mayor deterioro del nivel del producto y el incremento de la tasa de desempleo. En este marco, se esperaba que las citadas políticas dieran continuidad a la recuperación económica y revirtieran por completo en 2010 la ligera recesión de 2009, hecho que se confirmó a posteriori.

A continuación se detallan las medidas anticíclicas más importantes y la cronología del combate a la crisis $^{4}$. Las medidas se subdividen según los meses de su aplicación.

\section{Septiembre de 2008}

i) 19 de septiembre de 2008: cuatro días después de la quiebra de Lehman Brothers, considerada decisiva para que la crisis alcanzara niveles mundiales, el tipo de cambio brasileño sufre una depreciación del 5\%. Para contener la depreciación o, de acuerdo con Dornbusch (1976), evitar un intervencionismo excesivo (overshooting) en el tipo de cambio, el Banco Central del Brasil realiza una subasta de 500 millones de dólares;

ii) 24 de septiembre de 2008: el Banco Central del Brasil, mediante la circular $\mathrm{N}^{\mathrm{o}} 3.405$, aumenta de 100 millones de reales a 300 millones de reales el valor que

\footnotetext{
4 Véanse mayores detalles sobre las medidas adoptadas e incluso un análisis de aquellas más relevantes en el Relatório das Contas do Governo, ejercicio de 2009, en el sitio del Tribunal de Cuentas de la Unión.
} 
las instituciones financieras han de deducir del cálculo de la exigencia adicional sobre los recursos a la vista, depósitos a plazo y depósitos de ahorro. Además, prorroga la recaudación de los depósitos bancarios obligatorios sobre las operaciones de arrendamiento con opción de compra. La fecha para la entrada en funcionamiento de la tasa de recaudación del $25 \%$ sobre los recursos captados se pospone del 16 de enero al 13 de marzo de 2009;

\section{Octubre de 2008}

iii) $1^{\circ}$ de octubre de 2008: el Banco del Brasil, S.A. anticipa la liberación de crédito por un valor de 5.000 millones de reales para el financiamiento de la producción agrícola;

iv) 2 de octubre de 2008: el Banco Central del Brasil permite que los bancos reduzcan hasta un $40 \%$ los requisitos de reservas bancarias obligatorias, a condición de que compren operaciones de crédito de otras instituciones financieras. El objetivo, en este caso, era permitir la transferencia de carteras de bancos que presentaran problemas de liquidez;

v) 6 de octubre de 2008: la Presidencia de la República publica la medida provisoria $N^{\circ} 442$, que otorga poderes al Banco Central del Brasil para comprar carteras de bancos con sede en el país. En este caso, el objetivo era demostrar a los clientes de los bancos la intención del gobierno de defender los depósitos y de ese modo evitar corridas bancarias;

vi) 8 de octubre de 2008: en virtud de la pronunciada depreciación cambiaria, por sobre el 9\%, el Banco Central del Brasil decide vender dólares en el mercado a la vista;

vii) 9 de octubre de 2008: en la misma semana en que se publicó la medida provisoria que permitía al Banco Central del Brasil comprar carteras de bancos con problemas de liquidez, el Consejo Monetario Nacional (CMN) realiza su reglamentación;

viii) 13 de octubre de 2008: el Banco Central del Brasil aumenta el límite de deducción de los depósitos bancarios obligatorios para incrementar la liquidez de la economía;

ix) 14 de octubre de 2008: el Banco Central del Brasil reduce del $45 \%$ al $42 \%$ el porcentaje del cobro de los depósitos obligatorios y del encaje obligatorio aplicado a los recursos a la vista;

x) 16 de octubre de 2008: el Banco Central del Brasil permite la venta de activos entre los bancos para aumentar la disponibilidad de caja;

xi) 21 de octubre de 2008: se publica la medida provisoria $\mathrm{N}^{\circ} 443$ que autoriza al Banco del Brasil y a la Caja Económica Federal (CEF) para constituir subsidiarias $\mathrm{y}$ adquirir participación en instituciones financieras con sede en el Brasil; xii) 22 de octubre de 2008: el impuesto a las operaciones financieras (IOF) se reduce a 0 en las operaciones de liquidación de cambio referente a la entrada de inversiones en el Brasil, con el propósito de estimular la entrada de capitales y, de esa forma, reducir la presión sobre el mercado cambiario;

xiii) 27 de octubre de 2008: los bancos que adelantaran 60 contribuciones mensuales al Fondo de Garantía de Crédito podrían disminuir el respectivo valor del depósito obligatorio a la vista. Con ello se esperaba aumentar la liquidez de la economía;

xiv) 30 de octubre de 2008: el Sistema de la Reserva Federal (banco central estadounidense) y el Banco Central del Brasil establecen una línea de canje financiero (swap) por la suma de 30.000 millones de dólares, con vencimiento al 30 de abril de 2009. El objetivo era mejorar la liquidez del sistema financiero internacional;

xv) 30 de octubre de 2008: el Banco Central del Brasil modifica la remuneración del depósito obligatorio a plazo con la finalidad de acrecentar el crédito en el mercado;

\section{Noviembre de 2008}

xvi) 4 de noviembre de 2008: el Banco Central del Brasil modifica las reglas de las subastas de dólares para financiar las exportaciones. Visto el mencionado congelamiento del mercado de crédito comercial internacional, el banco central actuó para otorgar crédito a los exportadores;

xvii) 6 de noviembre de 2008: el Gobierno Federal libera créditos por una suma de 6.900 millones de dólares para pequeñas y medianas empresas y para el sector automotor;

xviii) 11 de noviembre de 2008: el gobierno estadual de São Paulo establece una línea de crédito de 4.000 millones de reales para el sector automotor. El mismo día, la CEF amplía el límite de préstamos para la compra de materiales de construcción de 7.000 reales a 25.000 reales;

xix) 12 de noviembre de 2008: la CEF libera una línea de crédito para personas físicas por 2.000 millones de reales, crédito orientado a la compra de electrodomésticos, equipos electrónicos, muebles y materiales de construcción;

xx) 13 de noviembre de 2008: desde el 1 de diciembre de 2008, la recaudación obligatoria adicional sobre los depósitos a la vista, a plazo y de ahorro se efectuaría a partir de títulos públicos. De ese modo se ampliaba la capacidad de financiamiento del sector público;

xxi) 14 de noviembre de 2008: la Presidencia de la República publica la medida provisoria $N^{\circ} 447$, que 
extiende en 10 días el plazo de recaudación del impuesto sobre los productos industrializados (IPI), el impuesto retenido en la fuente y la contribución a la seguridad social. El plazo de recaudación del Programa de Integración Social y de la Contribución para el Financiamiento de la Seguridad Social (PIS/COFINS) también se extendió cinco días. Se estimaba que el cambio en la fecha de recaudación de los impuestos contribuiría con 21.000 millones de reales a la caja de las empresas;

xxii) 21 de noviembre de 2008: se edita el decreto que rebaja el impuesto a las operaciones financieras (IOF) del 3,38\% al 0,38\% en los financiamientos de motocicletas, motonetas y ciclomotores;

\section{Diciembre de 2008}

xxiii) 11 de diciembre de 2008: mediante el decreto $\mathrm{N}^{\mathrm{o}} 6.687$ se redujeron los porcentajes del IPI aplicados a la venta de vehículos y camiones. Esa medida estaría vigente del 15 de diciembre de 2008 al 31 de marzo de 2009;

xxiv) 11 de diciembre de 2008: mediante el decreto $\mathrm{N}^{\mathrm{o}} 6.691$ se rebajaron los porcentajes del IOF aplicados a las operaciones de crédito con personas físicas;

xxv) 15 de diciembre de 2008: el Gobierno Federal, por medio de la medida provisoria $\mathrm{N}^{\mathrm{o}} 451$, anuncia la modificación de los porcentajes del impuesto sobre la renta de las personas físicas (IRPF); y

\section{Enero de 2009}

xxvi) 22 de enero de 2009: se edita la medida provisoria $\mathrm{N}^{\circ} 453$, que constituye una fuente adicional de recursos para el Banco de Desarrollo del Brasil (BNDES) por un valor de 100.000 millones de reales, cuyo objetivo era aumentar la disponibilidad de crédito de largo plazo.

La implementación de medidas anticíclicas continuó en el año 2009. Entre ellas se destaca la reducción de los porcentajes del IPI aplicados a los electrodomésticos de la línea blanca. También se exoneraron temporalmente del IPI los bienes de capital y materiales de construcción. Asimismo, se adoptaron otras medidas con respecto al crédito, entre ellas la reducción de la tasa de interés de largo plazo. Además, el gobierno adoptó una combinación de políticas de inversión y generación de ingresos. En el primer caso, se puede citar la creación del Programa de Aceleración del Crecimiento (PAC II), mientras que desde el punto de vista social es importante mencionar la política de construcción civil, representada por el programa Mi Casa Mi Vida, y el incremento de los recursos a disposición de la población mediante el aumento del valor del programa Bolsa Familia y del salario mínimo.

Si bien el detalle de las medidas de combate a la crisis y su respectiva cronología pueden parecer aburridos, el objetivo de este trabajo es analizar las medidas anticíclicas y su eficacia para aminorar los efectos adversos de la crisis internacional; por ello se considera que la extensa lista presentada es relevante para visualizar el camino tomado por el gobierno para combatir la crisis.

En resumen, las políticas aplicadas por el gobierno en la lucha contra la crisis se implementaron en diversas áreas. En primer lugar, se procuró mantener el sector externo mediante la concesión de crédito a los exportadores, dado que las líneas tradicionales estaban congeladas. Con respecto al sistema financiero, se tomaron medidas para mantener la liquidez y la solvencia de los bancos, al tiempo en que se señaló a los mercados la seria intención del gobierno de evitar una posible corrida bancaria. En cuanto a la reactivación de la economía, se puso en marcha una política monetaria expansionista mediante la reducción de las tasas de interés básicas y la ingente expansión del crédito. La política fiscal también fue expansionista a partir de la concesión de una serie de exenciones fiscales. 


\section{III}

\section{Eficacia de las medidas anticíclicas en el Brasil: análisis agregado}

En esta sección se aborda uno de los aspectos fundamentales de este trabajo: la eficacia de las políticas anticíclicas puestas en práctica por las autoridades brasileñas a fines de 2008 e inicios de 2009. Con este fin se examinan las medidas de política económica y se establece cuáles de ellas resultaron de hecho adecuadas para combatir la desaceleración de la economía y el aumento del desempleo, y evitar los efectos de la profundización de la crisis internacional en el Brasil. Se analizan entonces las medidas de política económica agregadas cuyo objetivo era producir efectos en la economía como un todo.

Acto seguido, se presentan pruebas empíricas de los efectos de las políticas anticíclicas en la economía brasileña. Sobre todo, se examinan los efectos de las políticas fiscal, monetaria y crediticia en el consumo de las familias, la inversión agregada y la brecha del producto.

\section{Efectos anticíclicos de las políticas monetaria y crediticia en el consumo de las familias}

A continuación se evalúan las repercusiones del crédito destinado al sector privado en el consumo agregado de las familias. En este análisis se toma como punto de partida la función de consumo keynesiana, que se extenderá para incorporar la variable crédito, de modo que:

$$
\text { Cons }_{t}^{\text {fam }}=\beta_{o}+\beta_{1} Y_{t}+\beta_{2} \operatorname{Cred}_{t}^{\text {pri }}
$$

Al normalizar la ecuación (1) mediante el ingreso agregado, se obtiene la siguiente función:

$$
\operatorname{Cons}_{t}^{\text {fam }} / Y_{t}=\beta_{o}+\beta_{1} \operatorname{Cred}_{t}^{\text {pri }} / Y_{t}
$$

Se espera un signo positivo del coeficiente $\beta_{1}$ relativo al crédito. Es decir, dado un determinado incremento en la relación entre el volumen de crédito al sector privado y el ingreso agregado se espera un aumento de la relación consumo de las familias por el mismo ingreso agregado.

Para eliminar la posibilidad de una regresión espuria, se optó por el uso de las variables definidas en la ecuación (2) en forma de tasas de variación. En el cuadro A.2 del anexo se demuestra que las variables consumo de las familias y crédito al sector privado - ambas sobre el ingreso- son estacionarias, de modo que se rechaza la hipótesis de raíz unitaria. La ecuación (2) puede entonces reescribirse en la forma estocástica. Además, se agregan dos variables, una interactiva y la variable ficticia $D 1$.

$$
\begin{gathered}
d\left(\operatorname{Cons}_{t}^{\text {fam }} / Y_{t}\right)=\beta_{0}+\beta_{1} * d\left(\operatorname{Cred}_{t}^{\text {pri }} / Y_{t}\right) \\
+\beta_{2} * d\left(\operatorname{Cred}_{t}^{\text {pri }} / Y_{t}\right) * D 2+\beta_{3} * D 1+u_{t}
\end{gathered}
$$

La variable $D 1$ se utiliza para captar los efectos anteriores al Plan Real, que - como se ha visto- corresponden a un período caracterizado por el descontrol inflacionario. Por otra parte, la variable interactiva, $d\left(\operatorname{Cred}_{t}^{\text {pri }} / Y_{t}\right)^{*} D 2$, se utiliza para evaluar los efectos de la variación de la relación entre crédito e ingresos a partir de la crisis de las hipotecas de alto riesgo - es decir, desde el primer trimestre de 2007 hasta el primer trimestre de 2010 (dummy D2) - en la variable depen-

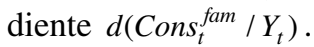

El parámetro estimado $\hat{\beta}_{1}$ proporcionará el efecto de la variable explicativa $d\left(\operatorname{Cred}_{t}^{\text {pri }} / Y_{t}\right)$ en la variable dependiente $d\left(\right.$ Cons $\left._{t}^{\text {fam }} / Y_{t}\right)$ en el período total. Por otra parte, la suma de los parámetros estimados $\hat{\beta}_{1}$ y $\hat{\beta}_{2}$ representará el efecto de la variable $d\left(\operatorname{Cred}_{t}^{\text {pri }} / Y_{t}\right)$ en la variable $d\left(\right.$ Cons $\left._{t}^{\mathrm{fam}} / Y_{t}\right)$ en el período comprendido entre enero de 2007 y enero de 2010. La suma de los parámetros estimados $\left(\hat{\beta}_{1}+\hat{\beta}_{2}\right)$ revelará el efecto de la variable de interés - en este caso el efecto conjunto de las variables $d\left(\mathrm{Cred}_{t}^{\text {pri }} / Y_{t}\right)$ y $d\left(\mathrm{Cred}^{\text {pri }} / Y\right) * D 2$ - en la variable dependiente $d\left(\right.$ Cons $\left._{t}^{\text {fam }} / Y_{t}\right)$ durante el período de la crisis. De esa forma, es posible evaluar las repercusiones de las políticas anticíclicas implementadas por el gobierno durante la crisis financiera. Este procedimiento se aplicará también a las demás estimaciones.

Los resultados indicados en el cuadro 1 muestran la estimación de la ecuación (3), con la que básicamente se analiza la manera en que el crédito, en sus diversas versiones, afectó al consumo de las familias. En primer lugar, se observa que todos los parámetros estimados son estadísticamente significativos al nivel de confianza del $95 \%$, excepto el parámetro relativo al intercepto $\left(\hat{\beta}_{0}\right)$. 
CUADRO 1

Estimación de la ecuación (3): efecto del crédito en el consumo de las familias

(De enero de 1991 a enero de 2010)

\begin{tabular}{|c|c|c|c|c|}
\hline Variable & Coeficiente & Desviación estándar & Estadística t-student & Estadística P \\
\hline Intercepto & $<0,001$ & 0,004 & 0,275 & 0,784 \\
\hline$d\left(\right.$ Credpri $\left.^{\prime} Y\right)$ & 0,608 & 0,049 & 12,272 & $<0,001$ \\
\hline$d(\text { Credpri } / Y)^{2} D 2$ & $-0,513$ & 0,181 & $-2,839$ & 0,006 \\
\hline D1 & $-0,023$ & 0,010 & $-2,261$ & 0,027 \\
\hline $\operatorname{AR}(1)$ & $-0,333$ & 0,087 & $-3,820$ & $<0,001$ \\
\hline $\mathrm{AR}(3)$ & $-0,644$ & 0,089 & $-7,215$ & $<0,001$ \\
\hline $\mathrm{MA}(2)$ & $-0,226$ & 0,094 & $-2,417$ & 0,018 \\
\hline MA(3) & 0,726 & 0,086 & 8,416 & $<0,001$ \\
\hline MA(4) & 0,428 & 0,077 & 5,540 & $<0,001$ \\
\hline $\mathrm{R} 2$ & 0,787 & & Estadística F & 29,648 \\
\hline Durbin-Watson (DW) & 1,903 & & $\begin{array}{l}\text { Estadística P } \\
\text { (Estadística F) }\end{array}$ & $<0,001$ \\
\hline
\end{tabular}

Fuente: elaboración propia.

Nota: ecuación estimada con el método de mínimos cuadrados ordinarios (MCO).

De acuerdo con las estadísticas detalladas en el cuadro 1, un incremento del $10 \%$ en la variable crédito, designada mediante $d\left(\mathrm{Cred}^{\text {pri }} / Y\right)$, supone un aumento de cerca del $6,1 \%$ en el consumo de las familias, $d\left(\right.$ Cons $\left._{t}^{\text {fam }} / Y_{t}\right)$. En otras palabras, el crédito produce realmente un efecto en la demanda agregada por la vía del consumo en el período analizado.

La suma de los parámetros estimados $\hat{\beta}_{1}$ y $\hat{\beta}_{2}$ produce un resultado positivo de 0,095 . Dicho resultado muestra que un incremento del $10 \%$ en el crédito, $d\left(\mathrm{Cred}^{p r i} / Y\right)$, supone un aumento de cerca del $1 \%$ en el consumo de las familias, $d\left(\right.$ Cons $\left._{t}^{\text {fam }} / Y_{t}\right)$, en el período de crisis. Esto revela que la política crediticia anticíclica implementada por el gobierno para combatir los efectos negativos de los desequilibrios externos incidió positivamente en el consumo de las familias.

Como se esperaba, la variable ficticia $D 1$ tuvo un efecto negativo en la variable consumo de las familias, lo que sugiere que el período de inestabilidad de precios anterior al Plan Real disminuía el poder de compra de las familias y, en consecuencia, afectaba negativamente a su consumo final. Sin limitarse a los efectos de la crisis financiera de 2008 y 2009, se puede concluir que ese resultado de la variable ficticia $D 1$ es bastante interesante, pues se llega efectivamente a la conclusión de que el descontrol de precios actuaba como un impuesto inflacionario sobre la sociedad, afectando negativamente al consumo final ${ }^{5}$.

\footnotetext{
${ }^{5}$ Se debe considerar que la regresión fue ajustada sobre la base de un modelo autorregresivo de media móvil (ARMA) de manera que los residuos se convirtieran en un proceso de ruido blanco. Los coeficientes estimados del AR(1), AR(3), MA(2), MA(3) y MA(4) son
}

Continuando el análisis de los efectos de las políticas anticíclicas monetaria y crediticia, se modificó la ecuación (3) para incorporar la tasa de interés real y se obtuvo la ecuación (4).

$$
\begin{aligned}
& d\left(\text { Cons }_{t}^{\text {fam }} / Y_{t}\right)=\beta_{0}+\beta_{1} * d\left(\text { Cred }_{t}^{\text {pri }} / Y_{t}\right) \\
& \quad+\beta_{2} * d\left(\text { Cred }_{t}^{\text {pri }} / Y_{t}\right) * D 2 \\
& \quad+\beta_{3} * d\left(r_{t-1}\right)+\beta_{4} * d\left(r_{t-1}\right) * D 2+u_{t}
\end{aligned}
$$

Para los efectos de simplificación, las variables en forma de tasa - como la tasa de variación de la relación entre el crédito y el ingreso y la tasa de variación de la tasa de interés real- se denominan crédito y tasa de interés real. Este mismo procedimiento se adopta a lo largo de todo el trabajo. Los resultados de la estimación de la ecuación (4) se detallan en el cuadro 2.

Las estadísticas presentadas en el cuadro 2 muestran que los parámetros estimados $\hat{\beta}_{1}$ y $\hat{\beta}_{3}$ son estadísticamente significativos al nivel de confianza del $95 \%$. Por otra parte, el parámetro $\hat{\beta}_{2}$ es marginalmente significativo al nivel de significancia del $10 \%$. De estos resultados se deduce que un incremento del $10 \%$ en la variable crédito, $d\left(\mathrm{Cred}^{p r i} / Y\right)$, supone un aumento de alrededor del 3,5\% en el consumo final de las familias, $d\left(\right.$ Cons $\left._{t}^{\text {fam }} / Y_{t}\right)$. La suma de los parámetros estimados $\hat{\beta}_{1}$ y $\hat{\beta}_{2}$ es 0,155 . Eso significa que un acrecentamiento del $10 \%$ en el crédito produce un incremento del $1,5 \%$

estadísticamente significativos al nivel del 5\%. Con esto se procura reafirmar la validez de la regresión. El correlograma de los residuos fue verificado y reveló un proceso de ruido blanco. 
Estimación de la ecuación (4): efecto del crédito y de la tasa de interés real en el consumo de las familias

(De enero de 1991 a enero de 2010)

\begin{tabular}{|c|c|c|c|c|}
\hline Variable & Coeficiente & Desviación estándar & Estadística t-student & Estadística P \\
\hline Intercepto & $-0,0005$ & 0,002 & $-0,222$ & 0,824 \\
\hline$d\left(C r e d^{p r i} / Y\right)$ & 0,355 & 0,066 & 5,397 & $<0,001$ \\
\hline$d(C$ redpri $/ Y) * D 2$ & $-0,200$ & 0,117 & $-1,709$ & 0,087 \\
\hline$d\left(r_{t-1}\right)$ & $-0,005$ & 0,002 & $-2,089$ & 0,037 \\
\hline$d\left(r_{t-1}\right) * D 2$ & 0,006 & 0,007 & 0,861 & 0,389 \\
\hline $\mathrm{AR}(1)$ & $-0,339$ & 0,076 & $-4,444$ & $<0,001$ \\
\hline $\mathrm{AR}(4)$ & 0,605 & 0,068 & 8,819 & $<0,001$ \\
\hline $\operatorname{MA}(2)$ & $-0,353$ & 0,100 & $-3,517$ & $<0,001$ \\
\hline $\mathrm{R} 2$ & 0,710 & & Estadística F & 14,689 \\
\hline Durbin-Watson (DW) & 2,103 & & $\begin{array}{c}\text { Estadística P } \\
(\text { Estadística F) }\end{array}$ & $<0,001$ \\
\hline
\end{tabular}

Fuente: elaboración propia.

Nota: ecuación estimada con el método de mínimos cuadrados ordinarios (MCO).

en el consumo de las familias durante el período de la crisis. Los parámetros estimados refuerzan la tesis de que la política crediticia adoptada, cuyo objetivo era mitigar los efectos de la crisis mundial en el Brasil, fue efectiva pues incidió positivamente en el consumo de las familias. Dichas estimaciones corroboran las detalladas en el cuadro $1^{6}$.

Se puede observar también que una reducción del $1 \%$ en la variable $d\left(r_{t-1}\right)$, representativa de la tasa de interés real, supone un incremento del $0,005 \%$ en el consumo final de las familias en todo el período analizado. El coeficiente estimado de la variable interactiva $d\left(r_{t-1}\right) * D 2$ es estadísticamente nulo. Eso significa que la variación de la tasa de interés real durante el período de crisis no afectó a la variación del consumo de las familias, manteniéndose el mismo coeficiente de $-0,005$. Una explicación para ese resultado podría ser que - al adquirir bienes de consumo duraderos- el consumidor prioriza como variable de control el plazo de financiamiento en lugar de la tasa de interés ${ }^{7}$, de modo que las prestaciones sean compatibles con su disponibilidad de ingresos 8 .

\footnotetext{
${ }^{6}$ Por otra parte, la inclusión de la tasa de interés real determinó la pérdida de significancia estadística de la variable $D 1$, que se refiere al período inflacionario previo al Plan Real, y en consecuencia fue descartada del modelo.

${ }^{7} \mathrm{El}$ aspecto fundamental para el consumidor no serían los intereses pagados sino la adecuación de la prestación a su presupuesto.

${ }^{8}$ En este caso la regresión también fue ajustada sobre la base de un proceso ARMA, de modo que los residuos se transformaran en ruido blanco. Los parámetros estimados del $\mathrm{AR}(1), \mathrm{AR}(4)$ y $\mathrm{MA}(2)$ son estadísticamente significativos al nivel del $1 \%$. Por último, el correlograma de los residuos verificó el proceso de ruido blanco.
}

\section{Efectos anticíclicos de las políticas monetaria y crediticia en la formación bruta de capital fijo (inversiones)}

En esta subsección se evalúan las repercusiones del crédito destinado al sector privado y de la tasa de interés real en la formación bruta de capital fijo (FBCF). Para el análisis se toma como punto de partida la función de inversión keynesiana ${ }^{9}$, que se amplía para incorporar la variable crédito.

$$
F B C F_{t}=\beta_{o}+\beta_{1} * Y_{t}+\beta_{2} * r_{t}+\beta_{3} * \operatorname{Cred}_{t}^{\text {pri }}
$$

Al normalizar la ecuación (5) mediante el ingreso agregado se obtiene la siguiente función:

$$
F B C F_{t} / Y_{t}=\beta_{o}+\beta_{1} * r_{t}+\beta_{2} *\left(\operatorname{Cred}_{t}^{p r i} / Y_{t}\right)
$$

Los resultados esperados son los coeficientes $\beta_{1} \mathrm{y}$ $\beta_{2}$, respectivamente negativo y positivo. De otra forma, se espera que la reducción de las tasas de interés reales estimule las inversiones, que también serían incentivadas por el aumento de las operaciones de crédito. Al transformar las variables en tasas de variación se obtiene la ecuación (7) ${ }^{10}$.

\footnotetext{
${ }^{9}$ Una versión completa de la función inversión keynesiana requeriría además la inclusión de una variable representativa de las expectativas empresariales.

10 Con esto se procura eliminar los riesgos de que la regresión sea espuria. La estacionariedad de las variables puede verse en el cuadro A.2 del anexo.
} 


$$
\begin{gathered}
d\left(\mathrm{FBCF}_{t} / Y_{t}\right)=\beta_{0}+\beta_{1} * d\left(r_{t}\right)+\beta_{2} * d\left(\mathrm{Cred}_{t}^{\text {pri }} / Y_{t}\right) \\
+\beta_{3} * d\left(\mathrm{Cred}_{t}^{\text {pri }} / Y_{t}\right) * D 2+\beta_{4} * d\left(r_{t}\right) * D 2+u_{t}
\end{gathered}
$$

Las estimaciones correspondientes a la ecuación (7) se detallan en el cuadro 3. Los resultados denotan que los parámetros estimados $\hat{\beta}_{0}, \hat{\beta}_{2}, \hat{\beta}_{3}$ y $\hat{\beta}_{4}$ no son estadísticamente significativos al nivel de confianza del 95\%. Estos resultados son reveladores, pues indican que la variación de la tasa de interés real no produjo efectos en la variación de la FBCF (inversión) en el análisis correspondiente a todo el período. Esto puede sugerir dos consecuencias: i) el principal factor determinante del nivel de inversión se relaciona con las expectativas de los empresarios, y ii) la tasa de interés que de hecho se utiliza para financiar una parte sustancial de la inversión del sector privado no es la tasa de interés básica de la economía o tasa SELIC ${ }^{11}$.

Otro resultado interesante se refiere al efecto estadísticamente nulo de los cambios de la política crediticia durante el período de crisis. Una vez más, la explicación de ese resultado puede estar vinculada al clima de pesimismo del empresariado. Así, las expectativas negativas con respecto al futuro de la economía contribuyeron a que los cambios en la política crediticia durante el período de crisis no se revelaran importantes para estimular nuevas inversiones. En el contexto de pesimismo debido la recesión mundial y, en menor medida, la nacional, los empresarios optaron por posponer las inversiones.

11 Las tasas relevantes serían las del BNDES y de otras instituciones gubernamentales de crédito.
Por otra parte, el coeficiente $\hat{\beta}_{1}$ es estadísticamente significativo al nivel de confianza del $95 \%$. Se deduce, en consecuencia, que el crédito es un factor determinante para las inversiones. Esa estimación puede interpretarse de la siguiente manera: un incremento del $10 \%$ en el crédito supone un crecimiento de las inversiones cercano a un $4 \%$ en el período total. Como ese parámetro no fue modificado en respuesta a la política crediticia expansionista en el período de crisis, $\hat{\beta}_{1}+\hat{\beta}_{2}=0,434$ $+0=0,434^{12}$.

\section{Efectos anticíclicos de la política fiscal en el producto}

Una vez evaluados los efectos de las operaciones de crédito y de la tasa de interés real en el consumo de las familias y la FBCF (inversiones), el análisis continúa y se amplía para medir no solo los efectos de las políticas monetaria y crediticia en el producto, sino también los de la política fiscal. La ecuación estimada se presenta a continuación.

$$
\begin{aligned}
& \text { Hiato }_{t}^{\text {PIB }}=\beta_{0}+\beta_{1} * \text { Hiato }_{t-1}^{r}+\beta_{2} * \text { Hiato }_{t-1}^{r} * D 2 \\
& +\beta_{3} * \text { Hiato }_{t-1}^{i m p / Y}+\beta_{4} * \text { Hiato }_{t-1}^{\text {imp } / Y} * D 2+\beta_{5} * \text { Hiato }_{t-1}^{\text {cred } / P} \\
& +\beta_{6} * \text { Hiato }_{t-1}^{\text {cred } / P} * D 2+\beta_{7} *(I M P / Y)^{2}+u_{t}
\end{aligned}
$$

Básicamente, en la ecuación (8) se aprecia que la brecha del producto, es decir, el diferencial entre el

\footnotetext{
${ }^{12}$ La regresión estimada se ajustó sobre la base de un proceso MA(24), de manera que los residuos presentaran un proceso de ruido blanco.

\begin{tabular}{|c|c|c|c|c|}
\hline Variable & Coeficiente & Desviación estándar & Estadística t-student & Estadística P \\
\hline Intercepto & 0,004 & 0,007 & 0,530 & 0,598 \\
\hline$d\left(\right.$ Credpri $\left.^{\prime} Y\right)$ & 0,434 & 0,188 & 2,313 & 0,024 \\
\hline$d\left(\right.$ Credpri $\left.^{\prime} Y\right) * D 2$ & $-0,487$ & 0,308 & $-1,582$ & 0,118 \\
\hline$d(r)$ & $-0,0007$ & 0,003 & $-0,190$ & 0,849 \\
\hline$d(r) * D 2$ & $-0,0002$ & 0,014 & $-0,011$ & 0,991 \\
\hline MA(24) & 0,911 & 0,035 & 26,188 & $<0,001$ \\
\hline $\mathrm{R} 2$ & 0,724 & & Estadística F & 36,789 \\
\hline Durbin-Watson (DW) & 1,971 & & $\begin{array}{l}\text { Estadística P } \\
\text { (Estadística F) }\end{array}$ & $<0,001$ \\
\hline
\end{tabular}
Se garantizó así la validez de la regresión estimada.
}

Estimación de la ecuación (7): efecto del crédito y de la tasa de interés real en la FBCF (De enero de 1991 a enero de 2010)

Fuente: elaboración propia.

Nota: ecuación estimada con el método de mínimos cuadrados ordinarios (MCO).

FBCF: ecuación formación bruta de capital fijo. 
producto y el producto potencial, resulta afectada por las brechas de la tasa de interés y de las operaciones de crédito - diferencia de la variable con respecto a su tendencia-, así como por la brecha tributaria. Las estimaciones referentes a la ecuación (8) se presentan en el cuadro 4.

De los resultados que aparecen en el cuadro 4 surge que los parámetros estimados son estadísticamente significativos al nivel de confianza del 95\%, excepto los parámetros $\hat{\beta}_{0}, \hat{\beta}_{3}, \hat{\beta}_{4}, \hat{\beta}_{7}$. Se constata que los impuestos, en sus diversas especificaciones, no afectaron en forma significativa a la brecha del producto y, en consecuencia, no sirvieron para mitigar los efectos de la crisis mundial de 2008 y 2009. Se observa que el coeficiente estimado del cuadrado de la relación impuestos/PIB tampoco es estadísticamente significativo, sugiriendo de esa forma la inexistencia de una relación no lineal entre la relación impuestos/PIB y la brecha del producto.

Por otra parte, el coeficiente de la tasa de interés presentó el signo negativo esperado, de modo que una reducción del 10\% en la variable desfasada referente a la tasa de interés supone un aumento del 0,05\% en la brecha del producto. Otra conclusión atañe al hecho de que ese resultado es idéntico a la relación entre la variación de la tasa de interés real y la variación del consumo de las familias. Si bien el coeficiente $\hat{\beta}_{1}$ es estadísticamente diferente de 0 , es sin embargo muy pequeño, lo que demuestra la baja elasticidad de la brecha del producto ante las variaciones de las tasas de interés.
Durante el período de crisis se observa un efecto negativo mayor de la brecha de la tasa de interés real en la brecha del producto. La suma de los parámetros estimados $\hat{\beta}_{1}$ y $\hat{\beta}_{2}$ produce un resultado negativo de $-0,05$. Eso significa que una reducción del $10 \%$ en la tasa de interés en el período de la crisis supone una mejora del $5 \%$ en la brecha del producto. Este resultado deja ver que la política monetaria expansionista produjo un efecto significativo en el proceso de recuperación de la economía brasileña. Al comparar el período normal con el período de crisis, se aprecia que la potencia del efecto de la tasa de interés aumentó un $1.000 \%$ específicamente en el período de crisis.

La brecha tributaria sobre el ingreso resulta marginalmente significativa al nivel del $10 \%$. En este caso se puede concluir que una reducción del $10 \%$ en la variable brecha tributaria desfasada supone un aumento cercano al $3 \%$ en la variable brecha del producto. Por otra parte, la variable interactiva Hiato ${ }_{t-1}^{i m p / Y} * D 2$ no es estadísticamente significativa. El resultado indica que la política fiscal anticíclica no fue estadísticamente significativa para explicar la recuperación de la economía brasileña durante el período de crisis.

En cuanto a la política crediticia, el modelo estimado a partir de la ecuación (8) presentó coeficientes significativos. En detalle, los coeficientes estimados con respecto al crédito - tanto el que cubre todo el período analizado como el relativo al lapso posterior a la crisis de las hipotecas de alto riesgo- afectan

CUADRO 4

Estimación de la ecuación (8): efectos de la brecha de la tasa de interés, de las operaciones de crédito y de la brecha tributaria en la brecha del producto (De enero de 1991 a enero de 2010)

\begin{tabular}{|c|c|c|c|c|}
\hline Variable & Coeficiente & Desviación estándar & Estadística t & Estadística P \\
\hline Intercepto & 0,093 & 0,064 & 1,462 & 0,149 \\
\hline Hiato $_{t-1}^{r}$ & $-0,005$ & 0,003 & $-2,009$ & 0,049 \\
\hline Hiato $_{t-1}^{r} * D 2$ & $-0,045$ & 0,017 & $-2,547$ & 0,013 \\
\hline Hiato $_{t-1}^{i m p / Y}$ & $-0,305$ & 0,169 & $-1,804$ & 0,076 \\
\hline Hiato omp $_{t-1} / Y * D 2$ & $-0,289$ & 0,372 & $-0,777$ & 0,440 \\
\hline Hiato $_{t-1}^{\text {cred } / P}$ & $2,48 \mathrm{E}-07$ & 8,78E-08 & 2,828 & 0,006 \\
\hline Hiatot $_{t-1}^{\text {cred } / P} * D 2$ & $-2,19 \mathrm{E}-07$ & $1,09 \mathrm{E}-07$ & $-2,007$ & 0,049 \\
\hline$\left(I M P_{t} / Y_{t}\right)^{2}$ & $-3,103961$ & 2,206929 & $-1,406$ & 0,164 \\
\hline $\mathrm{AR}(4)$ & 0,410 & 0,112 & 3,656 & $<0,001$ \\
\hline R2 & 0,542 & & Estadística F & 9,314 \\
\hline DW & 1,511 & & $\begin{array}{l}\text { Estadística P } \\
\text { (Estadística F) }\end{array}$ & $<0,001$ \\
\hline
\end{tabular}

Fuente: elaboración propia.

Nota: ecuación estimada con el método de mínimos cuadrados ordinarios (MCO). 
estadísticamente a la brecha del producto. No obstante, los resultados obtenidos corresponden a valores próximos a 0 . Esto conduce a concluir que el crédito, en la especificación dada por la ecuación (8), aparentemente afectó poco a la brecha del producto. En forma análoga, la suma de los parámetros $\hat{\beta}_{5}$ y $\hat{\beta}_{6}$ produjo una estadística significativa y con el signo esperado (positivo), pero con un valor cercano a 0 . Sin embargo, cabe destacar que los valores de la variable crédito están expresados en miles de millones de reales. De esa manera, la repercusión de un valor muy pequeño, pero estadísticamente diferente de 0 , en un valor muy elevado no es despreciable. Por consiguiente, y a partir del modelo especificado por la ecuación (8), se puede afirmar que el crédito produjo un efecto relevante en la brecha del producto ${ }^{13}$.

13 Una vez más la regresión fue ajustada. Se estimó un AR(4), que fue estadísticamente significativo al nivel del $1 \%$. De esa forma,
En virtud de lo expuesto y sobre la base de los resultados resumidos en el cuadro 5, se puede inferir que: i) el consumo de las familias respondió de forma positiva a la política crediticia expansionista; ii) la FBCF (inversión) no fue sensible a las políticas expansionistas monetaria y crediticia, y iii) el producto fue sensible a la política monetaria expansionista implementada mediante la reducción de las tasas de interés real y respondió positivamente a la expansión del crédito. Por último, cabe destacar que la política fiscal expansionista (que supuso la reducción de impuestos) no produjo efectos en el producto. Esta afirmación se corrobora al evaluar la restricción intertemporal del gobierno. Básicamente, la renuncia a la recaudación o los mayores gastos actuales deberán compensarse en un futuro próximo con medidas restrictivas que garanticen la solvencia de las cuentas públicas.

los residuos de la regresión ajustada presentaron un proceso de ruido blanco.

CUADRO 5

Resumen de los resultados de las políticas anticíclicas

\begin{tabular}{lccc}
\hline Variable dependiente & Política monetaria & Política crediticia & Política fiscal \\
\hline Consumo de las familias & Ineficaz & Eficaz & No se evaluó \\
Inversión & Ineficaz & Ineficaz & No se evaluó \\
Producto & Eficaz & Eficaz & Ineficaz \\
\hline
\end{tabular}

Fuente: elaboración propia.

\section{IV}

\section{Conclusiones}

El análisis empírico reveló que la política crediticia expansionista fue determinante para aumentar el consumo de las familias y el producto agregado durante la crisis. La política monetaria expansionista también fue importante para incrementar el producto agregado en ese mismo período. Se subraya que los gastos relativos a las inversiones no resultaron sensibles a las políticas anticíclicas monetaria, crediticia y fiscal. Dicho resultado puede explicarse por el elevado grado de incertidumbre y las expectativas negativas en cuanto al futuro de la economía, que posiblemente fueron decisivos para que los empresarios adoptaran una postura más cautelosa y conservadora, suspendieran gran parte de las inversiones en curso y cancelaran nuevos proyectos. Así, desde el punto de vista de la demanda agregada, las políticas monetaria y crediticia desempeñaron un papel relevante en la recuperación de la economía brasileña. Como nota discordante, se observa que la política fiscal resultó ineficaz para reactivar los negocios en el país.

Cabe destacar algunas consideraciones sobre la relación costo-beneficio de las políticas anticíclicas implementadas. Los resultados empíricos muestran - grosso modo - que al contrario de las políticas monetaria y crediticia, la política fiscal no fue gravitante en la recuperación del nivel de actividad económica. Además 
de brindar posiblemente un beneficio mínimo, la política fiscal supuso un costo elevado, debido al incremento de la deuda pública como resultado del mayor déficit fiscal nominal. Se sugiere estudiar las consecuencias relativas a la utilización de políticas fiscales expansionistas en futuras investigaciones, visto que - como se desprende de los análisis empíricos- el beneficio a corto plazo fue mínimo, pero el costo, incluso a corto plazo, no fue despreciable. Desde una perspectiva de largo plazo, el aumento de los gastos, sobre todo de aquellos de carácter permanente (salarios y previsión social, entre otros), y la reducción de la recaudación exigirán medidas adicionales para recuperar el ajuste fiscal, que podrán disminuir los recursos necesarios para la inversión privada.

ANEXO

CUADRO A.

Descripción de las variables agregadas

\begin{tabular}{lcc}
\hline Variable & Sigla & Unidad de medida \\
\hline $\begin{array}{l}\text { Consumo final de las familias } \\
\text { Producto interno bruto (PIB) a precios básicos }\end{array}$ & $\begin{array}{c}\text { Cons fam } \\
\text { Tasa básica de interés operada por medio del Sistema Especial }\end{array}$ & $\begin{array}{l}\text { Millones de reales } \\
\text { Millones de reales } \\
\text { de Liquidación y Custodia (SELIC) }\end{array}$ \\
$\begin{array}{l}\text { Formación bruta de capital fijo } \\
\begin{array}{l}\text { PIB real a precios de mercado deflactado por el Índice General } \\
\text { de Precios-Disponibilidades Internas (IGP-DI) }\end{array}\end{array}$ & $\begin{array}{l}\text { Porcentajes } \\
\text { Tasa de inflación según el Índice Nacional de Precios al }\end{array}$ & Millones de reales \\
$\begin{array}{l}\text { Consumidor Amplio (IPCA) } \\
\text { Crédito al sector privado }\end{array}$ & Millones de reales \\
$\begin{array}{l}\text { Impuestos sobre los productos } \\
\text { IPEA }\end{array}$ & Porcentajes \\
\hline
\end{tabular}

Fuente: elaboración propia.

Nota: Instituto de Investigación Económica Aplicada (IPEA).

CUADRO A.2

Prueba de raíz unitaria

(De enero de 1991 a enero de 2010)

\begin{tabular}{|c|c|c|c|c|c|c|}
\hline Variables & $\begin{array}{l}\text { Prueba de Dickey-Fuller } \\
\text { aumentada (ADF) (SIC) }\end{array}$ & $\begin{array}{l}\mathrm{ADF} \\
(\mathrm{SIC})\end{array}$ & $\begin{array}{l}\mathrm{ADF} \\
(\mathrm{SIC})\end{array}$ & $\begin{array}{c}\text { Prueba de } \\
\text { Phillips-Perron }\end{array}$ & $\begin{array}{c}\text { Prueba de } \\
\text { Phillips-Perron }\end{array}$ & $\begin{array}{c}\text { Prueba de } \\
\text { Phillips-Perron }\end{array}$ \\
\hline & $\begin{array}{c}\text { Valor } \\
\text { crítico } 5 \%\end{array}$ & $\begin{array}{l}\text { Estadístico } \\
\text { t-student }\end{array}$ & $\begin{array}{c}\text { Valor } \\
\text { estadístico P }\end{array}$ & $\begin{array}{c}\text { Valor } \\
\text { crítico } 5 \%\end{array}$ & $\begin{array}{l}\text { Estadístico } \\
\text { t-student }\end{array}$ & $\begin{array}{c}\text { Valor } \\
\text { estadístico P }\end{array}$ \\
\hline$(I M P / Y)^{2}$ & $-3,471 * *$ & $-2,861$ & 0,181 & $-3,470 * *$ & $-4,614$ & 0,002 \\
\hline$d(r)$ & $-1,946^{*}$ & $-6,563$ & $<0,001$ & $-1,945 *$ & $-9,206$ & $<0,001$ \\
\hline$d\left(\right.$ Cons $\left._{t}^{\text {fam }} / Y_{t}\right)$ & $-1,946 *$ & $-4,945$ & $<0,001$ & $-1,945^{*}$ & $-29,860$ & $<0,001$ \\
\hline$d\left(\right.$ Cred $\left._{t}^{p r i} / Y_{t}\right)$ & $-1,945^{*}$ & $-8,115$ & $<0,001$ & $-1,945^{*}$ & $-8,143$ & $<0,001$ \\
\hline Hiato $^{\text {cred }}$ & $-1,945^{*}$ & $-3,203$ & 0,002 & $-1,945^{*}$ & $-2,636$ & 0,009 \\
\hline Hiato $^{P I B}$ & $-1,946^{*}$ & $-2,196$ & 0,028 & $-1,945^{*}$ & $-5,728$ & $<0,001$ \\
\hline Hiato $^{\text {imp } / Y}$ & $-1,945^{*}$ & $-4,647$ & $<0,001$ & $-1,945^{*}$ & $-4,712$ & $<0,001$ \\
\hline Hiato $^{r}$ & $-1,945^{*}$ & $-8,743$ & $<0,001$ & $-1,945^{*}$ & $-10,310$ & $<0,001$ \\
\hline
\end{tabular}

Fuente: elaboración propia.

Notas: i) $\mathrm{H}_{0}$ : variable presenta raíz unitaria; ii) un asterisco (*) representa prueba con constante y dos asteriscos (**) prueba con constante y tendencia; y iii) $d x_{t}=\left(x_{t}-x_{t-1}\right) / x_{t-1}=\left(\Delta x_{t} / x_{t-1}\right)$.

SIC: criterio de información de Schwartz. 


\section{Bibliografía}

Banco Central del Brasil (varios números), Boletim do Banco Central do Brasil, Brasilia.

Dornbusch, Rudiger (1976), "Expectations and exchange rate dynamics", Journal of Political Economy, vol. 84, $\mathrm{N}^{\circ}$ 6, Chicago, University of Chicago Press.

Kacef, Osvaldo y Rafael López-Monti (2010), "América Latina, del auge a la crisis: desafíos de política macroeconómica", Revista de la CEPAL, $\mathrm{N}^{\circ} 100$ (LC/G.2442-P), Santiago de Chile.

Kindleberger, Charles P. y Robert Z. Aliber (2009), Da euforia ao pânico: uma história das crises financeiras, São Paulo, Editora Gente.

Krugman, Paul R. (2009), A crise de 2008 e a economia da depressão, Río de Janeiro, Elsevier.

Lopes, Francisco L. (2009), "A dimensão da crise", Como reagir à crise? Políticas econômicas para o Brasil, Edmar L. Bacha e Ilan Goldfajn (orgs.), Río de Janeiro, Imago.

Mohan, Rakesh (2009), "Global Financial Crisis: Causes, Impact, Policy Responses and Lessons", presentación en la Annual India Business Forum Conference, Londres, London Business School.

Rogoff, Kenneth S. y Carmem M. Reinhart (2010), Oito séculos de delírios financeiros: desta vez é diferente, Río de Janeiro, Elsevier.
Rose, Andrew K. y Mark M. Spiegel (2009), "Cross-country causes and consequences of the 2008 crisis: early warning", NBER Working Paper, $\mathrm{N}^{\mathrm{o}}$ 15357, Cambridge, Massachusetts, National Bureau Economic Research.

Roubini, Nouriel y Stephen Mihm (2010), A economia das crises: um curso-relâmpago sobre o futuro do sistema financeiro internacional, Río de Janeiro, Intrínseca.

Taylor, John B. (2009), "The financial crisis and the policy responses: an empirical analysis of what went wrong", NBER Working Paper, $\mathrm{N}^{\circ}$ 14631, Cambridge, Massachusetts, National Bureau Economic Research.

Torres Filho, Ernani Teixeira (2008), "Entendendo a crise do subprime", Visão do desenvolvimento, $\mathrm{N}^{\circ} 44$, Río de Janeiro, Banco de Desarrollo del Brasil.

Torres Filho, Ernani Teixeira y Gilberto Rodrigues Borça Júnior (2008), "A crise do subprime ainda não acabou", Visão do desenvolvimento, $\mathrm{N}^{\mathrm{o}}$ 50, Río de Janeiro, Banco de Desarrollo del Brasil.

TCU (Tribunal de Cuentas de la Unión) (2009), "Relatório das contas do governo. Exercício de 2009", [en línea] portal2.tcu.gov.br] 